\title{
LIGHT LEPTOQUARKS AND HIGGS PAIR PRODUCTION
}

\author{
Ts. Enkhbat \\ Institute of Physics and Technology, Mongolian Academy of Sciences, \\ Peace Avenue 54B, Ulaanbaatar 13330, Mongolia \\ E-mail:enkhbat@gmail.com
}

\section{INTRODUCTION}

The data collected by the LHC experiments at 7 and $8 \mathrm{TeV}$ with $\sim 5$ and $20 \mathrm{fb}^{-1}$ respectively is refining the details of the Higgs like resonances found last year [1,2]. Many decay channels have been searched for and the individual channels so far have given us a consistent picture with what one expects from the SM Higgs. On the other hand, the self interaction of the Higgs, which is probed by the Higgs pair production [3-7], is too feeble in the SM to be detected with these early data set. Even at $14 \mathrm{TeV}$ run, the luminosity required for probing this process is very high [7-17]. This fact, namely the smallness of the corresponding Higgs pair production crosssection, makes it sensitive to a presence of a new physics [18-31].

In particular, relatively light colored particles are known to affect the cross-section substantially [18-22]. As a mater of fact there are many models with various motivations including models of GUT remnants [3239], composite models [40-48] or a radiative neutrino mass models [49-51] which may give such contributions. Among these the scalars are interesting as they may play crucial role in the spontaneous symmetry breaking through additional terms with large portal couplings in the scalar potential.

In the present work we study the phenomenological consequences of the Standard Model extension by two or more colored scalar particles. As a case study we take several leptoquarks (LQ) since there is an active experimental program by both ATLAS and CMS [52-57] and the lower bounds on their masses have now reached impressive levels some as high as a $\mathrm{TeV}$ value. On the other hand simultaneous presence of several LQs, may open up additional channels and therefore weakens these bounds. Specific models where the LQs are introduced to explain a certain phenomenon usually requires more than one LQs as in the model we study here.

I examine a possibility of the existence of LQs with masses as light as $\sim 180 \mathrm{GeV}$ and study their effect for the single and di Higgs productions. As we will see the Higgs pair production is substantially altered in the low mass range below $300 \mathrm{GeV}$ without too much change in the Higss diphoton decay channel if portal couplings are large. These couplings are required to have opposite signs by the latest Higgs data or small in magnitude. The model I consider has two LQs, an SU(2) doublet $\omega$ and a singlet $\chi$. As we will see their simultaneous presence still allows them to have relatively light masses and escape the current bounds. In particular, the current bounds do not include LQs decaying to $\tau \mathrm{t}$ the masses below $200 \mathrm{GeV}$. Such a scenario, for example, has appeared in a model considered by Babu and Julio [49], where the light neutrino masses are induced by two-loop effects from LQs. If their masses are only of order few hundred $\mathrm{GeV}$, as it is required in this case, the scenario can be probed or even excluded with the data from the LHC. Therefore this is one of the easiest 
model which can be tested and is the subject of the current study. Although I consider a particular model, it should be stressed that other models with colored particles can affect the pair productions in a similar manner.

In Section II, I briefly list the current experimental status on the Higgs production

\section{LIGHT LEPTOQUARKS}

ATLAS and CMS both have released their results on the Higgs searches from 7 and $8 \mathrm{TeV}$ runs [58-60]. These results indicate that the diphoton and various other Higgs decay channels are consistent with the SM predictions. Therefore, any new resonance should not affect the single Higgs production and its various decay channels too much. This requirement alone makes a single colored scalar object harder to exist at lower mass range if its portal coupling of $|\mathrm{H}|^{2}|\mathrm{X}|^{2}$ type and decay rates. Then I introduce the model I examined in the paper. Section III contains main part of this work where the numerical results for the single and pair Higgs productions are presented.

The conclusion is given in Section IV.

$$
\begin{aligned}
& \mathcal{L}=\left(Y_{i j} \Omega i \sigma_{2} L_{i} d_{j}^{c}+F_{i j} \chi e_{i}^{c} u_{j}^{c}-\mu \Omega^{\dagger} H \chi+h . c\right)-m_{\Omega}^{2}|\Omega|^{2}-m_{\chi}^{2}|\chi|^{2} \\
& -\lambda_{\omega}|\Omega|^{2}|H|^{2}-\lambda_{\chi}|\chi|^{2}|H|^{2}-\kappa\left|\Omega^{\dagger} H\right|^{2}
\end{aligned}
$$

After the electroweak symmetry breaking the lower component of the doublet LQ will mix with the singlet $L Q$ via the trilinear is large. If such couplings are small they will not play any interesting role in the Higgs phenomenology. On the other hand several colored scalars can lead to interesting excesses that may be checked with the existing data at the same time satisfying various Higgs decay channels measurements.

The model I examine in this paper contains two new multiplets, $S U(2)_{L}$ singlet and doublet scalar leptoquarks $\Omega(3,2,1 / 6)$ and $\chi(3,1,-1 / 3)$ [49]. The Lagrangian of the model is given as:

$$
\begin{aligned}
& m_{\omega}^{2}=m_{\Omega}^{2}+\frac{\lambda_{\omega}}{2} v^{2}, \\
& m_{\chi_{1}, \chi_{2}}^{2}=\frac{1}{2}\left(m_{\omega}^{2}+\frac{\kappa}{2} v^{2}+m_{\chi}^{2}+\frac{\lambda_{\chi}}{2} v^{2} \mp \sqrt{m_{\omega}^{2}+\frac{\kappa}{2} v^{2}-m_{\chi}^{2}-\frac{\lambda_{\chi}}{2} v^{2}+2 \mu^{2} v^{2}}\right), \\
& \tan 2 \vartheta=\frac{2 \sqrt{2 \mu v}}{2 m_{\omega}^{2}+\kappa v^{2}-2 m_{\chi}^{2}-\lambda_{\chi} v^{2}},
\end{aligned}
$$

where $\vartheta$ and $m_{\chi_{1}, \chi_{2}}$ are the mixing angle and masses for the $-1 / 3$ charged LQs $\chi_{1}$ and $\chi_{2} . \mathrm{m}_{\omega}$ is the mass of $2 / 3$ charged component denoted as $\omega$. This spectrum was proposed by Babu and Julio as an explanation for the light neutrino masses induced by the two-loop effects of the LQs. Readers interested in are referred to the original paper where exhaustive list of many flavor implications were discussed. Several scenarios in the model require these LQs to be lighter than $500 \mathrm{GeV}$, which makes them testable at the LHC. I concentrate primarily on the portal couplings and study their collider aspect and examine the consequences.

The searches for LQs at LHC have given lower bounds on their masses for several 
different LQ decay channels for the data collected at $7 \mathrm{TeV}$ by both CMS and ATLAS collaborations [61-67]. These results show that the LQ are at least heavier than $500 \mathrm{GeV}$ or so if the branching fractions are assumed to be $100 \%$. On the other hand the search for the LQ decaying to tau and top $(\tau t)$, leaves the possibility of the LQ lighter than $200 \mathrm{GeV}$ [68]. This is because $\tau$ is not energetic enough to be identified efficiently. A thorough collider search analysis of this process in this mass range is beyond scope of this paper. Interested readers are referred to Refs [46, 69-73].

If one considers any of the LQs, the LHC searches require that their masses have to be above $450 \mathrm{GeV}$. Unless corresponding portal coupling is very large the both single and di Higgs productions will not be affected at any interesting level. In the following we explain that these constraints may not be applicable for the model given by the Lagrangian in Eq. (1), To do so we consider a case where the following mass hierarchy holds: $m_{\chi_{2}}>m_{\chi_{1}}$. If the couplings $Y_{i j}$ in Eq (1) are small enough such that the mass splitting between $2 / 3$ and $-1 / 3$ charged LQ makes $\omega \rightarrow \chi_{i} W^{+*} \rightarrow \chi_{i} \bar{f}_{d} f_{u}$ channel dominant, these bounds are evaded. We call these three-body channels. Here $\bar{f}_{d} f_{u}=(\bar{d} u, \bar{s} c, \bar{\ell} v)$. The star signifies that the $\mathrm{W}$ is off-shell. This is because the electroweak precision test requires the mass splitting within the $\mathrm{SU}(2)$ doublet components be less than $52 \mathrm{GeV}$ [73]. Among the $F_{i j}$ couplings $F_{33}$ is allowed to be large by the LQ searches since it leads to the not yet ruled-out $t \tau$ decay channel. Therefore we further assume the other $F_{i j}$ couplings are small and satisfy the constraints $[49,50,74,75]$. Further, if $F_{33}$ is the largest $F_{i j}$ coupling, $\chi \rightarrow \tau \bar{t}$ to be the dominant $\chi_{1}$ decay channel. From the above discussion we see that the signals for the $\omega$ pair production are $\chi_{i} \chi_{j} W^{+*} W^{-*}$ with the off shell W's subsequently decaying either hadronically or leptonically when $F_{33}$ is the largest coupling.

\section{HIGGS PHENOMENOLOGY WITH LIGHT LEPTOQUGRKS}

In the last section we have argued that the current limits from the LHC experiments still allow LQs with light masses down to $\sim 180 \mathrm{GeV}$ [67]. Previous study by the author explored the range to $300 \mathrm{GeV}$ [68]. Given that such a possibility exists in the current section we study their phenomenological consequences. The possibility that the Standard Model Higgs could have portal couplings to an unknown sector has been a subject of many studies due to its possible role in the electroweak symmetry breaking, electroweak phase transition and as the contact with the dark sector. Recent discovery of the SM Higgs like resonance has intensified such studies.
The effect we investigate here is the Higgs boson pair production. We take several LQs and choose large portal couplings to demonstrate the di-Higgs production rate can be dramatically increased while the single Higgs production and diphoton rates are affected within the experimentally measured values. This will happen even with the current data if the LQs are relatively light below 300 $\mathrm{GeV}$, which makes the model testable in most of the considered mass range. With the current constraint the not yet ruled out range 180 to 200 GeV. From the Lagrangian given in Eq.(1), the LQ and Higgs interactions are easily written down in the mass eigenstates as follows

$$
\begin{aligned}
V_{L Q-h}= & \left\{\left(\lambda_{\omega} c_{\vartheta}^{2}+\kappa c_{\vartheta}^{2}+\lambda_{\chi} s_{\vartheta}^{2}\right)\left|\chi_{1}\right|^{2}+\left(\lambda_{\omega} s_{\vartheta}^{2}+\kappa s_{\vartheta}^{2}+\lambda_{\chi} c_{\vartheta}^{2}\right)\left|\chi_{2}\right|^{2}+\lambda_{\omega} \omega^{2}\right. \\
& \left.+\left(\lambda_{\omega}+\kappa-\lambda_{\chi}\right) s_{\vartheta} c_{\vartheta}\left(\chi_{1} \chi_{2}^{*}+\chi_{2} \chi_{1}^{*}\right)\right\}\left(\frac{h^{2}}{2}+h v\right) \\
& +\left\{\mu \left(\left(\left|\chi_{2}\right|^{2}-\left|\chi_{1}\right|^{2} c_{\vartheta} s_{\vartheta}+\chi_{1}^{*} \chi_{2} c_{\vartheta}^{2}-\chi_{2}^{*} \chi_{1} s_{\vartheta}^{2}+h . c\right\} \frac{h}{\sqrt{2}},\right.\right.
\end{aligned}
$$

where $s_{\vartheta}\left(c_{\vartheta}\right) \equiv \sin \vartheta(\cos \vartheta)$. We choose the physical masses $m_{\omega, \chi_{(1,2)},}$ portal couplings $\lambda_{\omega}, \lambda_{\chi}$ 
and the mixing angle as the input parameters. Then the remaining parameters $\mu$ and $k$ are fixed through Eqs. (2-4). For the masses we take hierarchy $m_{\omega}>m_{\chi_{2}}>m_{\chi_{1}}$. In addition I choose $\Delta m \equiv m_{\omega}-m_{\chi_{2}}=10$ and $50 \mathrm{GeV}$ for small and large splitting and a constant value of $10 \mathrm{GeV}$ for the mass splitting between the lighter two $m_{\chi_{2}}-m_{\chi_{1}}=10 \mathrm{GeV}$. I take two different values for the LQ mixing $\sin \vartheta=0.1$ and $1 / \sqrt{2}$ for small and large mixings respectively.

Previous studies have considered an effect of a single colored particle, where one is forced to have a specific coupling not to upset the Higgs production rate. For example, the new physics contribution is chosen to be roughly twice larger and opposite in sign to have unaltered rate. This inevitably affects diphoton channel. In particular among possible color scalars only octet candidate was a good choice [20]. For these models, stability of vacuum requires increasingly stronger portal couplings as the mass is increased [76]. This is not required in our case, since we have several new contributions which can be kept under control by a judicious choices of the various portal.

We first scan over the $\lambda_{\omega}$ and $\lambda_{\chi}$ parameter space for the Higgs pair production and super impose the allowed regions by both CMS and ATLAS experiments by the diphoton and $\mathrm{ZZ}^{*}$ channels. The result is show in Figure 1. The lightest LQ mass is chosen to be $m_{\chi_{1}}=200$
$\mathrm{GeV}$. The parameter scan has been done using MadGraph 5 [77] with CTEQ6L1 PDF set [78]. The MadGraph implementation of the Higgs pair production in the SM has been modified to include contributions from the LQ. The code has been checked against previously known results such as in Ref [20] and was found to be in an excellent agreement.

As we can see there are regions in the parameter space where the single Higgs production and decay rates are compatible with either of CMS and ATLAS experiments. Depending on the values for the couplings the Higgs pair production may become substantially enhanced. The shape of the regions are easily understood. The single Higgs production rate and decay to diphoton and $\mathrm{ZZ}^{*}$ channels will be affected less if the contributions from the LQs largely cancel each other. This fact is reflected in the stripe regions. There is another possibility that the total LQ contribution is twice bigger than the SM amplitude and but opposite in sign as has been done in Ref $[20,21]$. This possibility is represented by the allowed region in the lower right corner of the scanned plots in Figure 1. where both $\lambda_{\omega}$ and $\lambda_{\chi}$ are large and negative. Since this region will be pushed to higher values as the LQ mass is increased we do not consider this region further and concentrate on the stripe regions. 

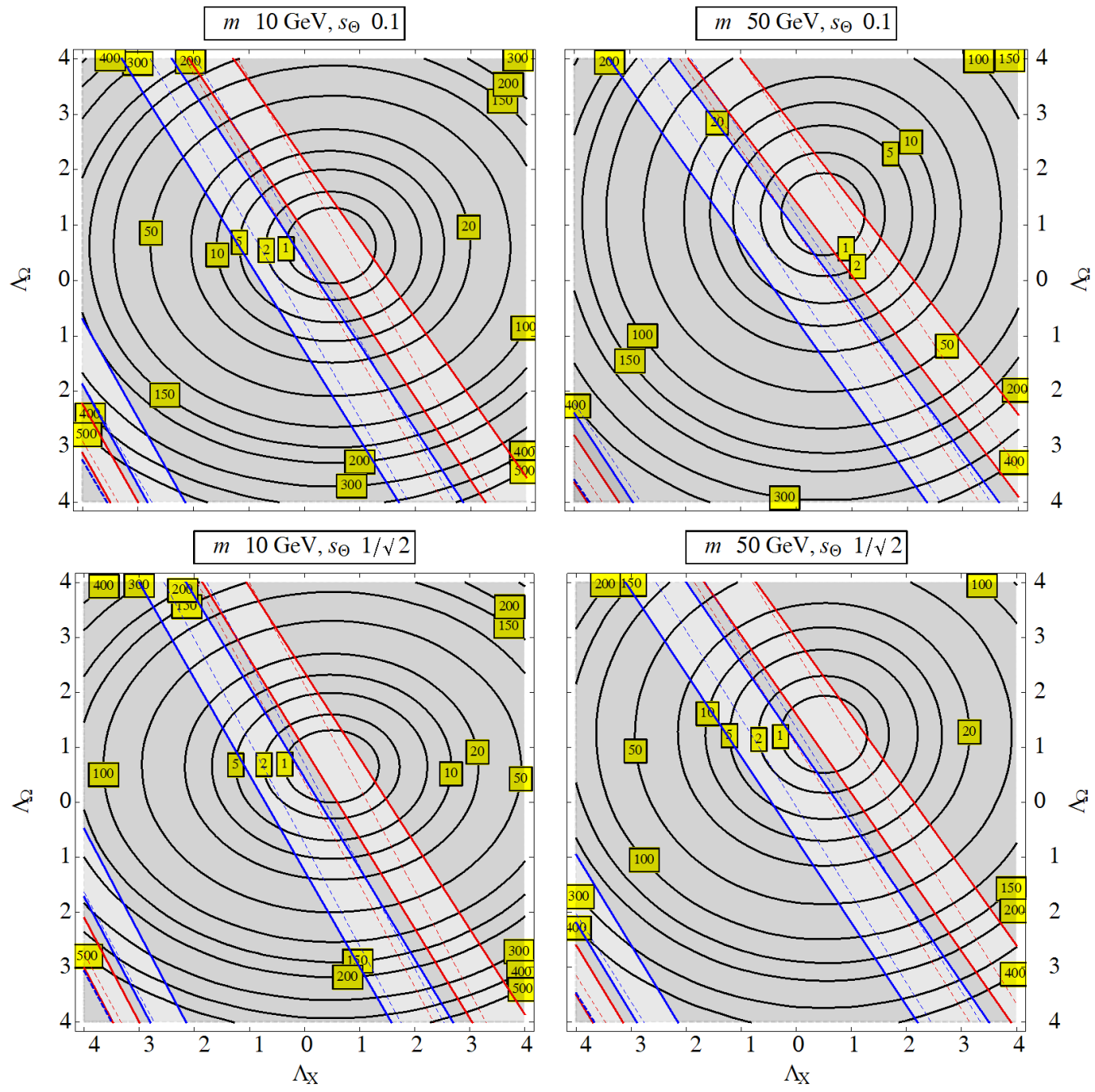

Figure 1. Scanned contour plot in - plane for the ratio Higgs pair productions due LQs and the SM. Here the mass of the lightest $L Q$ is chosen to be $200 \mathrm{GeV}$.

Next, I estimate the Higgs pair production for several set of and parameters. The results are shown in Figures 2 and 3. These are the main results of the present work. As we can see the rate may be enhanced quite substantially compared to the SM expectation even the single Higgs production is affected moderately. This is especially so in the range $180-200 \mathrm{GeV}$. The cancellation due to the opposite signs for and, which kept the single Higgs rate largely unchanged, is still operational for the triangular loop diagram contributions to the pair production. However, there are diagrams quadratic in the portal couplings whenever the final state Higgs legs come from different vertices. They will contribute constructively even if the single Higgs production remains the same as in the SM. The largest values I chose for the portal couplings require even larger value for the quartic couplings for LQ to make the vacuum at least metastable [68] since we have a negative portal coupling. If we generously allow and take values up to $4 \pi$ for the quartic couplings the metastability of the 
vacuum is guaranteed.

A detailed signal simulation for the LQ pair productions for the LHC experiments is beyond the scope of the present paper. Nevertheless, few comments are in order. The search for signal has recently done by CMS collaboration. The pair production cross- section is roughly an order of magnitude below that of if little above. Then the signal is hard to distinguished from as the taus would not be energetic enough. Therefore such light LQs are still a possibility. As for higher values starting around $200 \mathrm{GeV}$, this search rules out the leptoquarks decaying to $t \tau$.

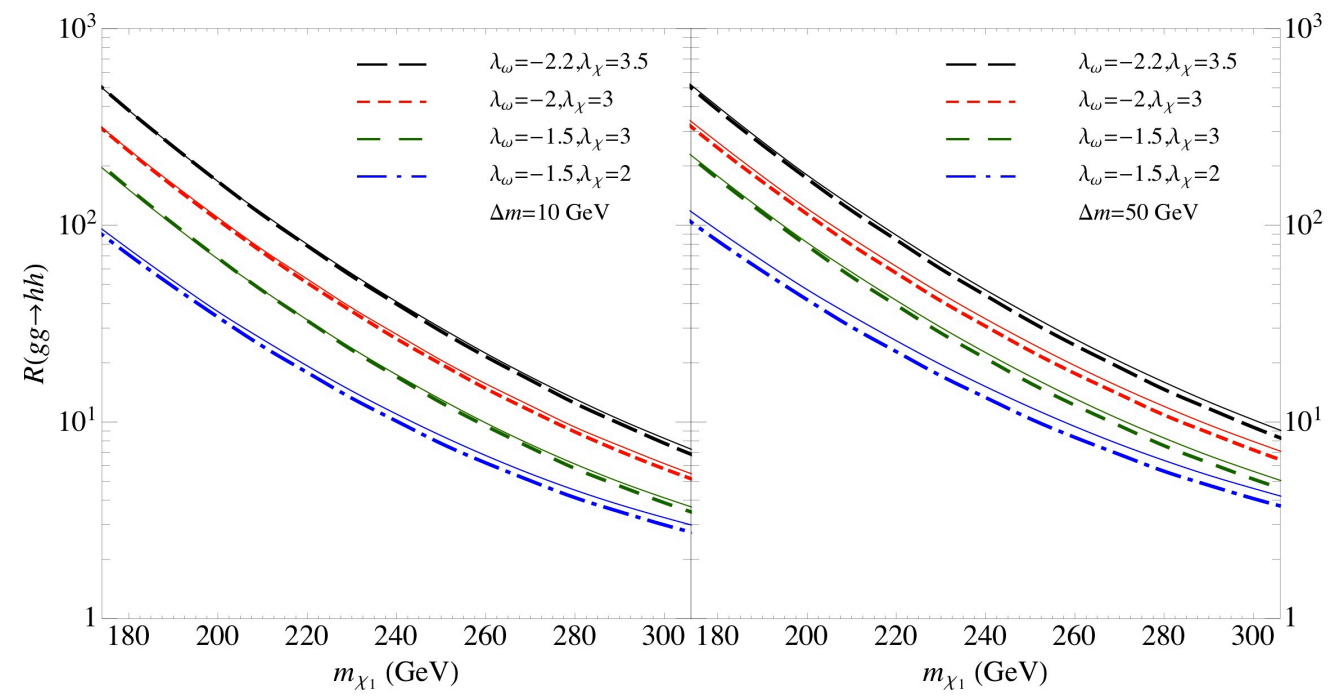

Figure 2. The ratio of Higgs productions due LQs and the SM for negative and positive for several different choices for the mixing parameter $=0.1$. The thin lines with the same colors to the various dashed lines are obtained when the maximal mixing = is chosen.

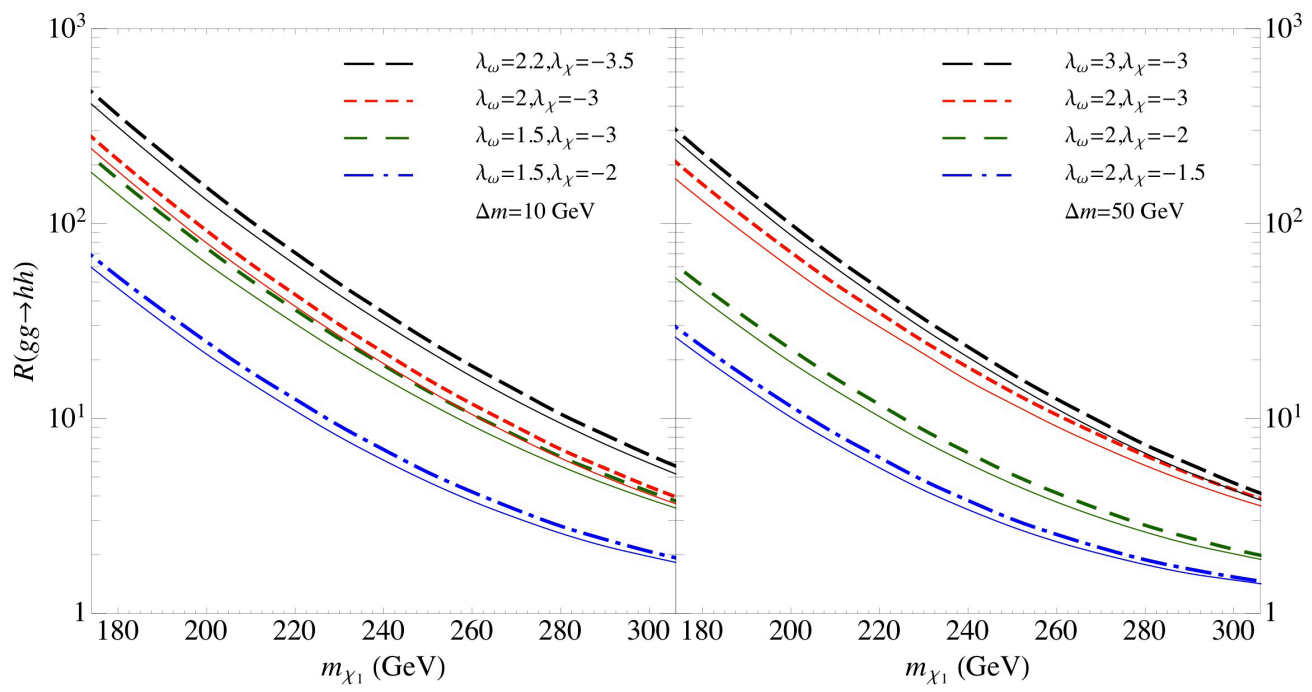

Figure 3. The ratio of Higgs pair productions due LQs and the SM for positive and negative. The solid (dashed) curves are for the LQ mixing angle with $=$. 


\section{CONCLUSIONS AND DISCUSSIONS}

The discovery of a scalar particle by CMS and ATLAS experiments at the LHC, which appears to be essentially consistent with the SM Higgs picture within experimental margin of error, is a triumph in our understanding of the fundamental dynamics. On the other hand, a firm confirmation itself is still far and many fundamental questions are left unanswered which can be addressed with new dynamics or particles at the TEV range.

In the present paper, I have considered a several scalar LQs in which their portal couplings are such that its effect on the single Higgs production is within the limits given by the either CMS or ATLAS experiment. Even in this case it has been found that the Higgs pair production can be modified substantially. For several set of values for the portal couplings it has been shown that the rate may reach one to two orders magnitude higher than what it is in the SM. The two portal couplings are chosen to have opposite signs which give reasonable single Higgs production rate via gluon fusion.

These are done via the following procedure.
Upon scanning over these couplings for a low mass value the allowed regions by the Higgs production and decay to diphoton and $\mathrm{ZZ}^{*}$ are obtained. Several set of values are chosen from these regions. For the chosen values the rates were found to be enhanced by various values. For the sets with larger values, it may reach two orders of magnitude at lower range of LQ masses with moderate effect on the single Higgs production. The effect becomes negligible above around the mass of 300 $\mathrm{GeV}$. For this value, the enhancements range from few to at most an order of magnitude. This makes the light mass region between 180 to $200 \mathrm{GeV}$ very intriguing where the enhancement for the Higgs pair production is most pronounced.

The present work demonstrates that the light colored particles with large portal couplings may reveal additional dynamics in the scalar potential. These are interesting due to their potential role in EWSB itself or in the thermal phase transition in the early universe.

\section{BIBLIOGRAPHY}

[1] G. Aad et al. [ATLAS Collaboration], Phys. Lett. B 716 (2012) 1

[2] S. Chatrchyan et al. [CMS Collaboration], Phys. Lett. B 716 (2012) 30.

[3] O. J. P. Eboli, G. C. Marques, S. F. Novaes and A. A. Natale, Phys. Lett. B 197, 269 (1987).

[4] E. W. N. Glover and J. J. van der Bij, Nucl. Phys. B 309, 282 (1988).

[5] D. A. Dicus, C. Kao and S. S. D. Willenbrock, Phys. Lett. B 203, 457 (1988).

[6] T. Plehn, M. Spira and P. M. Zerwas, Nucl. Phys. B 479, 46 (1996) [Erratum-ibid. B 531, 655 (1998)].

[7] S. Dawson, S. Dittmaier and M. Spira, Phys. Rev. D 58, 115012 (1998).

[8] A. Djouadi, W. Kilian, M. Muhlleitner and P. M. Zerwas, Eur. Phys. J. C 10, 45 (1999).

[9] S. Dittmaier et al., arXiv:1101.0593 [hep-ph].

[10] G. C. Branco et al., Phys. Rept. 516, 1 (2012).

[11] J. Baglio et al., JHEP 1304, 151 (2013).

[12] M. J. Dolan, C. Englert and M. Spannowsky, JHEP 1210, 112 (2012).

[13] A. Papaefstathiou, L. L. Yang and J. Zurita, Phys. Rev. D 87, 011301 (2013).

[14] F. Goertz, A. Papaefstathiou, L. L. Yang and J. Zurita, JHEP 1306, 016 (2013).

[15] D. de Florian and J. Mazzitelli, Phys. Lett. B 724, 306 (2013).

D. de Florian and J. Mazzitelli, Phys. Rev. Lett. 111, 201801 (2013).

[16] J. Grigo, J. Hoff, K. Melnikov and M. Steinhauser, Nucl. Phys. B 875, 1 (2013).

[17] V. Barger, L. L. Everett, C. B. Jackson and G. Shaughnessy, arXiv:1311.2931 [hep-ph].

[18] A. Belyaev et al., Phys. Rev. D 60, 075008 (1999). 
[19] E. Asakawa et al., Phys. Rev. D 82, 115002 (2010).

[20] G. D. Kribs and A. Martin, Phys. Rev. D 86, 095023 (2012).

[21] I. Dorsner, S. Fajfer, A. Greljo and J. F. Kamenik, JHEP 1211, 130 (2012).

[22] J. Cao, Z. Heng, L. Shang, P. Wan and J. M. Yang, JHEP 1304, 134 (2013).

Z. Heng, L. Shang and P. Wan, JHEP 1310, 047 (2013).

[23] C. Han, X. Ji, L. Wu, P. Wu and J. M. Yang, arXiv:1307.3790 [hep-ph].

[24] L. Wang, W. Wang, J. M. Yang and H. Zhang, Phys. Rev. D 76, 017702 (2007).

[25] X. -F. Han, L. Wang and J. M. Yang, Nucl. Phys. B 825, 222 (2010).

[26] M. J. Dolan, C. Englert and M. Spannowsky, Phys. Rev. D 87, no. 5, 055002 (2013).

[27] S. Hao and Z. Ya-Jin, JHEP 1211, 127 (2012).

[28] L. Wang and X. -F. Han, JHEP 1205, 088 (2012).

[29] D. Y. Shao, C. S. Li, H. T. Li and J. Wang, arXiv:1301.1245 [hep-ph].

[30] J. M. No and M. Ramsey-Musolf, arXiv:1310.6035 [hep-ph].

[31] N. Haba, K. Kaneta, Y. Mimura and E. Tsedenbaljir, arXiv:1311.0067 [hep-ph].

[32] H. Georgi and S. L. Glashow, Phys. Rev. Lett. 32, 438 (1974).

[33] J. C. Pati and A. Salam, Phys. Rev. D 10, 275 (1974) [Erratum-ibid. D 11, 703 (1975)].

[34] G. Senjanovic and A. Sokorac, Z. Phys. C 20, 255 (1983).

[35] O. U. Shanker, Nucl. Phys. B 204, 375 (1982).

[36] W. Buchmuller and D. Wyler, Phys. Lett. B 177, 377 (1986).

[37] W. Buchmuller, R. Ruckl and D. Wyler, Phys. Lett. B 191, 442 (1987) [Erratum-ibid. B 448, 320 (1999)].

[38] V. D. Angelopoulos et al.,Nucl. Phys. B 292, 59 (1987).

[39] J. L. Hewett and T. G. Rizzo, Phys. Rept. 183, 193 (1989).

[40] S. Dimopoulos and L. Susskind, Nucl. Phys. B 155, 237 (1979).

[41] S. Dimopoulos, Nucl. Phys. B 168, 69 (1980).

[42] E. Eichten and K. D. Lane, Phys. Lett. B 90, 125 (1980).

[43] E. Farhi and L. Susskind, Phys. Rept. 74, 277 (1981).

[44] B. Schrempp and F. Schrempp, Phys. Lett. B 153, 101 (1985).

[45] J. Wudka, Phys. Lett. B 167, 337 (1986).15

[46] B. Gripaios, JHEP 1002, 045 (2010).

[47] P. Q. Hung and C. Xiong, Nucl. Phys. B 847, 160 (2011).

[48] T. Enkhbat, W. -S. Hou and H. Yokoya, Phys. Rev. D 84 (2011) 094013.

[49] K. S. Babu and J. Julio, Nucl. Phys. B 841, 130 (2010).

[50] K. S. Babu and J. Julio, Phys. Rev. D 85, 073005 (2012).

[51] M. Kohda, H. Sugiyama and K. Tsumura, Phys. Lett. B 718, 1436 (2013).

[52] M. Pietroni, Nucl. Phys. B 402, 27 (1993).

[53] J. M. Cline and G. D. Moore, Phys. Rev. Lett. 81, 3315 (1998).

[54] J. M. Cline, G. D. Moore and G. Servant, Phys. Rev. D 60, 105035 (1999).

[55] M. Carena, G. Nardini, M. Quiros and C. E. M. Wagner, Nucl. Phys. B 812, 243 (2009).

[56] T. Cohen and A. Pierce, Phys. Rev. D 85, 033006 (2012)

[57] D. J. H. Chung, A. J. Long and L. -T. Wang, Phys. Rev. D 87, 023509 (2013).

[58] G. Aad et al. [ATLAS Collaboration], Phys. Lett. B 726, 88 (2013).

[59] CMS PAS HIG-13-001.

[60] CMS PAS HIG-13-002.

[61] S. Chatrchyan et al. [CMS Collaboration], Phys. Rev. D 86, 052013 (2012).

[62] CMS PAS EXO-12-042.

[63] S. Chatrchyan et al. [CMS Collaboration], JHEP 1212, 055 (2012).

[64] S. Chatrchyan et al. [CMS Collaboration], Phys. Rev. Lett. 110, 081801 (2013).

[65] G. Aad et al. [ATLAS Collaboration], JHEP 1306, 033 (2013).

[66] G. Aad et al. [ATLAS Collaboration], Eur. Phys. J. C 72, 2151 (2012).

[67] CMS-PAS-EXO-12-030.

[68] Ts. Enkhbat, JHEP 1401 (2014) 158. 
[69] M. Kramer, T. Plehn, M. Spira and P. M. Zerwas, Phys. Rev. D 71, 057503 (2005).

[70] A. Belyaev, C. Leroy, R. Mehdiyev and A. Pukhov, JHEP 0509, 005 (2005).

[71] B. Gripaios, A. Papaefstathiou, K. Sakurai and B. Webber, JHEP 1101, 156 (2011).

[72] S. Davidson and P. Verdier, Phys. Rev. D 83, 115016 (2011).

[73] J. Beringer et al. (Particle Data Group), Phys. Rev. D 86, 010001 (2012).

[74] S. Davidson, D. C. Bailey and B. A. Campbell, Z. Phys. C 61, 613 (1994).16

[75] S. Davidson and S. Descotes-Genon, JHEP 1011, 073 (2010).

[76] M. Reece, New J. Phys. 15, 043003 (2013).

[77] J. Alwall, M. Herquet, F. Maltoni, O. Mattelaer and T. Stelzer, JHEP 1106 (2011) 128.

[78] T. Sjostrand, S. Mrenna and P. Z. Skands, JHEP 0605 (2006) 026. 